Acta Crystallographica Section A

Foundations of Crystallography

ISSN 0108-7673

Received 13 April 2013

Accepted 2 July 2013

(C) 2013 International Union of Crystallography Printed in Singapore - all rights reserved

\section{Minimal nets and minimal minimal surfaces}

\author{
Liliana de Campo, ${ }^{\mathrm{a}}$ Olaf Delgado-Friedrichs, ${ }^{\mathrm{a}}$ Stephen T. Hyde ${ }^{\mathrm{a}}$ and Michael \\ $\mathbf{O}^{\prime}$ Keeffe $^{\mathbf{b}, \mathrm{c} *}$
}

\author{
${ }^{a}$ Department of Applied Mathematics, Research School of Physics, Australian National University, \\ Canberra, ACT 0200, Australia, ${ }^{\mathbf{b}}$ Department of Chemistry and Biochemistry, Arizona State \\ University, Tempe, AZ 85207, USA, and ${ }^{\mathbf{c}}$ Graduate School of EEWS(WCU), KAIST, 373-1, Guseng \\ Dong, Yuseong Gu, Daejeon 305-701, Republic of Korea. Correspondence e-mail: \\ mokeeffe@asu.edu
}

\begin{abstract}
The 3-periodic nets of genus 3 ('minimal nets') are reviewed and their symmetries re-examined. Although they are all crystallographic, seven of the 15 only have maximum-symmetry embeddings if some links are allowed to have zero length. The connection between the minimal nets and the genus- 3 zeromean-curvature surfaces ('minimal minimal' surfaces) is explored by determining the surface associated with a net that has a self-dual tiling. The fact that there are only five such surfaces but 15 minimal nets is rationalized by showing that all the minimal nets can serve as the labyrinth graph of one of the known minimal minimal surfaces.
\end{abstract}

\section{Introduction}

The special periodic nets known as minimal nets and the periodic surfaces known as minimal surfaces are of exceptional importance in the chemistry of crystalline materials. In particular minimal surfaces occur in materials on a variety of length scales and also play a key role in the systematic enumeration of periodic nets. In this paper we review what is known about them and derive some new properties that emphasize the close relationship between nets and surfaces.

The term net is used here to refer to a periodic simple connected graph. Minimal nets are those that have the minimal number of vertices and edges in their repeat units. More specifically, their quotient graphs (Chung et al., 1984) have cyclomatic number, $g$, equal to $d$ where $d$ is the periodicity of the net. In this paper we are concerned exclusively with 3-periodic structures, so $d=3$. It is elementary to show that if the quotient graph has $v$ vertices and $e$ edges, then $g=1$ $+e-v$. It is also well established that for $g=3$ there are exactly 15 minimal nets (Beukemann \& Klee, 1992). In the Reticular Chemistry Structure Resource (RCSR) database of nets (O'Keeffe et al., 2008), $g$ is identified as the genus of the net.

A surface has two principal curvatures $k_{1}$ and $k_{2}$ that are the maximum and minimum values. The mean curvature is $\left(k_{1}+\right.$ $\left.k_{2}\right) / 2$ and the Gaussian curvature is $K=k_{1} k_{2}$. 3-Periodic minimal surfaces (TPMSs) are those for which the mean curvature is everywhere zero. These may also be characterized by genus. A useful measure of their topology is offered by the genus of a volume of the surface, bounded by the primitive translational unit cell of the oriented TPMS (defined by three lattice vectors of the oriented surface whose two sides are colored distinctly, so that isometrics of the surface do not swap sides). This genus can be determined in a variety of ways (Hyde, 1989; Fischer \& Koch, 1989); it must again be at least 3 for 3-periodic nets (Meeks, 1977, 1990), so in what follows we call TPMSs of genus 3 minimal minimal surfaces (MMSs). Note that for a tiling of a surface in which the tiling has $v$ vertices, $e$ edges and $f$ faces (tiles) per repeat unit $f-e+v=2$ $-2 g$.

TPMSs play a central role in crystal chemistry and in materials science. Among the many TPMSs that have been explored in a mathematical context, it is clear that those of genus 3 are most relevant to the science of materials. Their enumeration is not entirely straightforward; at present five distinct MMSs are recognized. Four of these were described in the 19th century (Schwarz, 1890) but the fifth, the gyroid or $G$ surface, was not recognized until much later (Schoen, 1970, 2012). It has since been recognized that the gyroid is the most prevalent minimal surface in nature, found in natural and artificial materials, from chitin assemblies in butterfly wings, to liquid crystal structures, to atomic assemblies in microporous materials including mesoporous silicas (Hyde et al., 2008). Unlike the $P$ and $D$ surfaces, the $G$ surface contains neither straight lines in the surface [which are coincident with twofold axes of the (unoriented) TPMS] nor mirror planes. In the language of minimal surface theory, it is a Bonnet intermediate case, while the $P$ and $D$ examples are Bonnet endmembers. The $P$ and $D$ surfaces are also of some importance in materials science (Han et al., 2011).

The known MMSs are called balance surfaces because they divide space into two labyrinths that are either identical or (in the case of $G$ ) related as mirror images. The genus of the net of the channels in the labyrinth (the labyrinth graph) is the same as that of the surface (Fischer \& Koch, 1989; Hyde, 1989); accordingly the labyrinth graph must be one of the minimal 
nets. As there are 15 minimal nets, but only five known minimal minimal surfaces, the question arises as to whether there are other MMSs waiting discovery. Given the particular relevance of these surfaces to materials science, especially in mesoporous materials, it is disturbing to note that the catalog of them remains possibly incomplete.

A flat point of a TPMS is a point where the Gaussian curvature vanishes. The structure of the TPMS in the neighborhood of these flat points determines the flat-point order, $b$. The genus is related to the sum (over the unit cell) of flat-point orders $b_{i}$ by $g=1+\sum_{i} b_{i} / 4$ (Hyde, 1989). Meeks (1977) established that for all MMSs $b=1$, so these simplest TPMSs contain eight distinct isolated flat points.

The 'shape' of each genus-3 TPMS is governed locally by the surface normal vectors at these eight flat points. For example, the cubic $P$ and $D$ TPMSs contain eight flat points, oriented towards the eight $\langle 111\rangle$ directions. We note that, despite their locally identical structures, these two TPMSs are very different globally. Essentially, they differ in how their surface patches are stitched together to form periodic structures in space. To see that, consider wrapping identical twodimensional $4^{3} .6$ hyperbolic nets onto the $P, G$ and $D$ surfaces (Fig. 1). All give crystalline nets; however, the nets wrap to give distinct 'collars' around channels of these surfaces, resulting in different three-dimensional net topologies, namely rho (on the $P$ ), gie (on the $G$ ) and uks (on the $D$ ). The first two of these describe the framework topologies of zeolites with framework types RHO and BSV. We note that systematic generation of 3-periodic nets by projection of hyperbolic tilings onto 3-periodic Euclidean surfaces is the basis of the EPINET project (Ramsden et al., 2009).

These minimal surfaces admit lower-symmetry variants; indeed, Meeks (1977; see also Oguey, 1999) established that a five-parameter family of deformations is allowed for a generic (triclinic) genus-3 minimal surface. Explicit constructions of some lower-symmetry MMSs, including (five-parameter) triclinic genus-3 TPMSs, can be found in Fogden \& Hyde (1992). For example, the cubic $P$ and $D$ surfaces have a common rhombohedral MMS, the so-called $r P D$ surface. All the Bonnet end-members admit symmetry-lowering deformations that preserve the minimal surface geometry (Meeks, 1977). Rhombohedral and tetragonal variants of the $G$ have also been found (Fogden et al., 1993; Weyhaupt, 2008).

To summarize: the exploration of TPMSs that are Bonnet end-members is largely complete. That search yields generic triclinic TPMSs, capable of a (limited) range of deformations
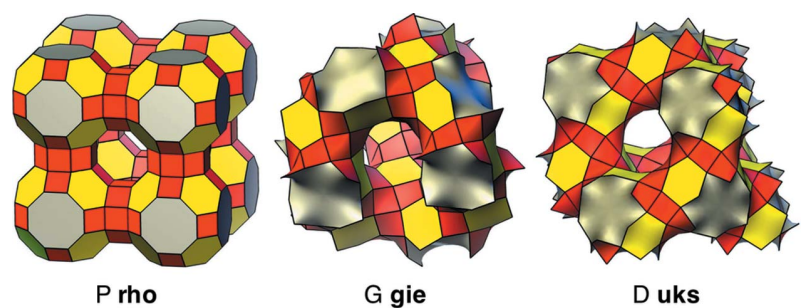

D uks of the triclinic lattice metric. All of these examples are symmetry-reduced variations of the five most symmetric genus-3 TPMSs, the $P, D, C L P$ and $H$ surfaces typical examples show hybrid character, such as the $r P D$ surfaces, and the (monoclinic) $m P C L P$ surface (Fogden \& Hyde, 1992, 1999). In contrast, however, Bonnet intermediate surfaces such as $G$ are far less well understood (Weyhaupt, 2008). The completeness or otherwise of the enumeration is one of the questions addressed in this paper.

It proves valuable first to re-examine the symmetries of embeddings of the minimal nets. This leads us to recognize an important class of crystallographic nets in which certain nodes coincide in a maximum-symmetry embedding.

\section{Minimal nets and their embeddings}

It is convenient to distinguish between abstract nets and their Euclidean embeddings (Chung et al., 1984). Specifically the vertices and edges of the graph correspond in this paper to nodes and kinks in an embedding. One reason for doing this is that we want to refer to the length of a link, whereas the 'length' of an edge has no meaning.

In an equilibrium (or barycentric) placement each vertex is assigned coordinates that are the mean of the coordinates of its neighbors. If the coordinates of one vertex are chosen (e.g. as $0,0,0)$ those of the others are uniquely determined. This provides a convenient identification of vertices and edges that allows determination of symmetry (Delgado-Friedrich \& O'Keeffe, 2003), but a placement is not an embedding as no metric is assigned. Nets for which, in an equilibrium placement, the vertices have distinct coordinates have a combinatorial symmetry group that is isomorphic with a space group and have embeddings in that space group (DelgadoFriedrichs, 2005). In what follows we refer to that space group as 'the symmetry of the net'.

Eight of the minimal nets have distinct barycentric coordinates for all vertices and have been assigned RCSR symbols; their embeddings, natural tilings and other properties have been described (Bonneau et al., 2004). These eight fall into two subgroups.

There are five 'parent' graphs with symbols pcu, dia, srs, cds, hms. These are recognized as the labyrinth graphs of the known MMSs $P, D, G, C L P, H$, respectively. In the embeddings of these nets, for the unit cell to have nonzero volume all links must have nonzero edge length. There is a second set of three, with symbols ths, tfa, tfc, that were not previously associated with minimal surfaces. We note that for this set some links can be made arbitrarily short or even of zero length with finite unit-cell volume. We discuss the surfaces associated with these three nets below.

Quotient graphs of the minimal nets are shown in Figs. 2 and 3 in a way that is meant to be suggestive. In the figures the edges corresponding to links that must have nonzero length are shown in black. The edges corresponding to links that can be zero are shown in blue. Note that, if these edges are made of zero 'length' and the vertices they join subsumed in one vertex, we recover the parent graph.

$4^{3} .6$ tilings of the $P, G$ and $D$ surfaces. 


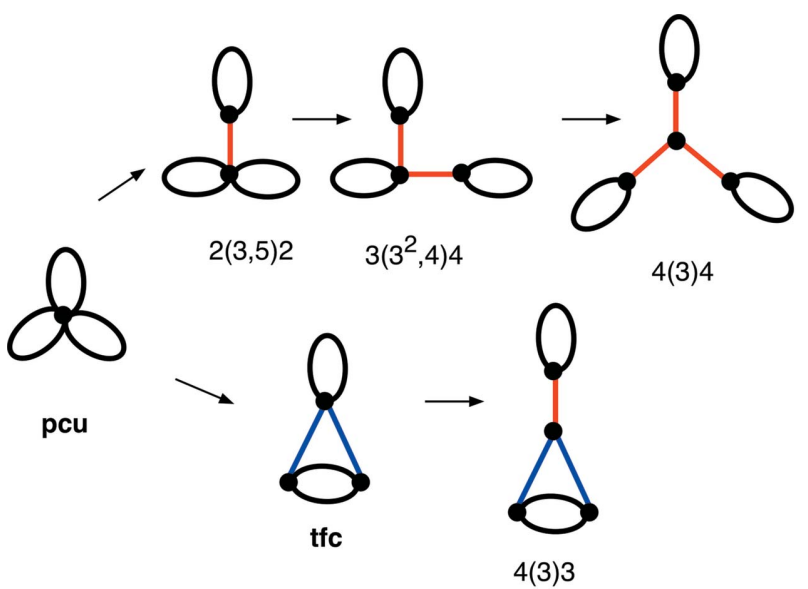

Figure 2

Five quotient graphs of minimal nets 'derived' from pcu. Bridges are shown in red. Blue edges correspond to links that can have zero length (see text). Symbols other than three-letter RCSR symbols are those of Beukemann \& Klee (1992).

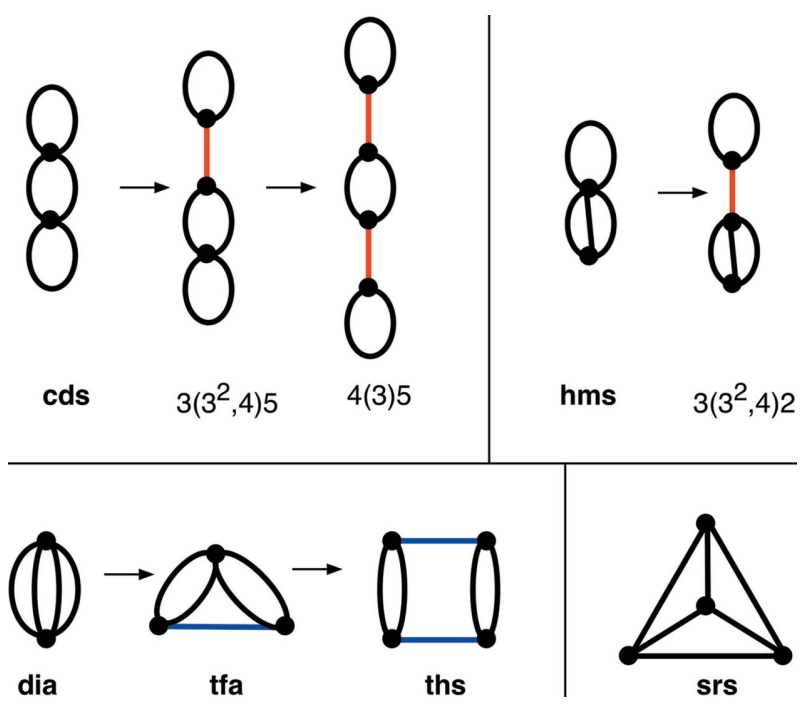

Figure 3

The nine quotient graphs of minimal nets not shown in Fig. 2. Bridges are shown in red. Blue edges correspond to links that can have zero length (see text). Each box contains a 'parent' graph and its derived graphs. Symbols other than three-letter RCSR symbols are those of Beukemann \& Klee (1992).

There remain seven minimal nets, for which we use the symbols assigned by Beukemann \& Klee (1992), whose quotient graphs have bridges (or cut edges). A bridge in a graph is an edge that joins two otherwise disjoint parts. For these graphs, pairs of vertices joined by bridges have the same coordinates in an equilibrium placement. However, such vertices can be distinguished in these cases as they have different sets of edges and again a symmetry, isomorphic to a space group, can be determined (Eon, 2007). However, the only possible embeddings in that symmetry require the links connecting nodes corresponding to vertices with identical barycentric coordinates to have zero length. The edges corresponding to these links are shown in red in Figs. 2 and 3. The symmetries of these graphs (Table 1) appear not to have
Table 1

The symmetry of the minimal nets with collisions.

The shape symmetry is the symmetry of the pattern of finite links in a maximum-symmetry embedding. In parentheses is the surface with which this shape corresponds to the labyrinth graph. The maximum symmetry is the graph symmetry. The finite-link symmetry is the maximum symmetry for an embedding with finite link length as reported by Eon (2011).

\begin{tabular}{llll}
\hline Net & Shape symmetry & $\begin{array}{l}\text { Maximum } \\
\text { symmetry }\end{array}$ & $\begin{array}{l}\text { Finite-link } \\
\text { symmetry }\end{array}$ \\
\hline $4(3) 4$ & $P m \overline{3} m(P)$ & $P m \overline{3} m$ & $R 32$ \\
$3\left(3^{2}, 4\right) 4$ & $P m \overline{3} m(P)$ & $P m m m$ & $C 2$ \\
$2(3,5) 2$ & $P m \overline{3} m(P)$ & $P 4 / m m m$ & $C m m 2$ \\
$4(3) 5$ & $P 4_{2} / m m c(C L P)$ & $P 4_{2} / m m c$ & $A m a 2$ \\
$3\left(3^{2}, 4\right) 5$ & $P 4_{2} / m m c(C L P)$ & $P m m m$ & $P m m 2$ \\
$4(3) 3$ & $C m m m(P)$ & $C m m m$ & $C m m 2$ \\
$3\left(3^{2}, 4\right) 2$ & $P \overline{6} m 2(H)$ & $P \overline{6} m 2$ & $C m m 2$ \\
\hline
\end{tabular}

been given explicitly before, and embeddings of these nets have previously been given in subgroups that allow finite links (Beukemann \& Klee, 1992; Eon, 2011). These graphs are derived from pcu, cds, hms and tfe and are shown in Figs. 4-6 with the ideally zero-length links shown as short black links. It should be clear that when those links really are of zero length, the remaining links form patterns identical ${ }^{1}$ to those of pcu, tff, cds and hms, and thus could also be considered as the labyrinth graphs of the $P, P C L P$ and $H$ surfaces, respectively. Eon (2011) has given the maximum possible symmetry for an embedding with links of finite length. These are compared with the full symmetry of the graph in Table 1.

Many, but not all, nets admit a tiling. By a tiling we mean a division of space into finite generalized polyhedra or cages that are topological spheres and which fill space when packed together face-to face. In a dual tiling new vertices are placed in the center of the original tiles and joined by new edges that pass through the faces of the original tiles. Noting that the dual of the dual is the original tiling completes the definition. A proper tiling of a net is one for which the symmetry of the tiling is the same as the symmetry of the net.

For the 'parent' nets that are identified above as the labyrinth graphs of the known MMSs, there is a unique proper tiling that has the property of being self-dual. The other three nets without collisions (i.e. without vertices with identical barycentric coordinates, ths, tfa, tfe) have two proper tilings; in each case one of them is self-dual. ${ }^{2}$ In $\S 3$ we show how tilings derived from these self-dual tilings illustrate the surface for which these nets are labyrinth graphs. The two nets, of the tiling and the dual, are related by symmetry so the assembly of two (denoted by - $\mathbf{c}$ in the RCSR) has a higher symmetry. The symmetries of a single net and the pairs of nets are listed in Table 2.

\footnotetext{
${ }^{1}$ Grünbaum (2003) has suggested the term isomeghethic for structures with the same pattern of nodes and links.

${ }^{2}$ For nets with more than one proper tiling, rules have been devised to determine a unique natural tiling (Delgado-Friedrichs et al., 2003; Blatov et al., 2007). One rule is that in a natural tiling no tile should have one face larger than the rest. The self-dual tilings for tfc and ths violate this rule. The natural tiling for tfa is self-dual. This last statement corrects an error in Bonneau et al. (2004).
} 
Table 2

Symmetries of the minimal nets without collisions and of an interpenetrating pair of those nets.

\begin{tabular}{lll}
\hline Net (surface) & Single net & Pair of nets \\
\hline pcu $(P)$ & $P m \overline{3} m$ & $I m \overline{3} m$ \\
srs $(G)$ & $I 4_{1} 32$ & $I a \overline{3} d$ \\
dia $(D)$ & $F d \overline{3} m$ & $P n \overline{3} m$ \\
cds $(C L P)$ & $P 4_{2} / m m c$ & $P 4_{2} / m c m$ \\
hms $(H)$ & $P \overline{6} m 2$ & $P 6_{3} / m m c$ \\
ths & $I 4_{1} / a m d$ & $P 4_{2} / n n m$ \\
tfa & $I \overline{4} m 2$ & $I 4_{1} / a m d$ \\
tfc & $C m m m$ & $F m m m$ \\
\hline
\end{tabular}

We have not found tilings for the seven minimal nets with collisions, but we note that the absence of a tiling can be difficult to prove.

\section{Surfaces associated with nets}

Since the work of Schoen (1970), the relation between TPMSs and 3-periodic nets has been recognized. Schoen described the $P, D$ and $G$ surfaces in terms of their 'skeletal nets', what we now call 'labyrinth graphs', which follow the channels of these surfaces. These graphs are the genus-3 pcu $(P)$, dia $(D)$ and srs $(G)$ nets. Two more minimal nets describe the non-cubic Bonnet end-members [hms $(H)$ and cds $(C L P)]$.

It should be recognized, however, that the concept of a labyrinth graph, while almost self-evident for the most
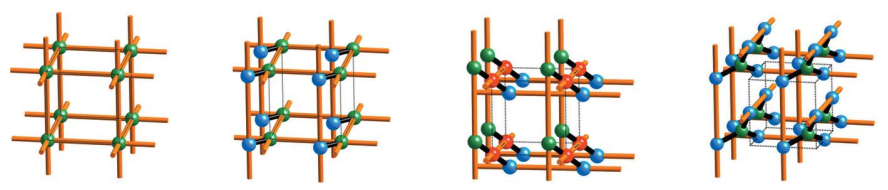

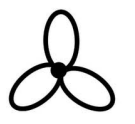

pcu

$P m-3 m$

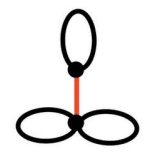

$2(3,5) 2$

$\mathrm{P} 4 / \mathrm{mmm}$

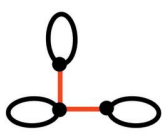

$3\left(3^{2}, 4\right) 4$

Prmm

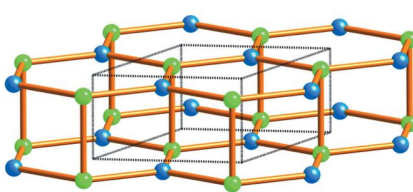

0

hms, $P-6 m 2$

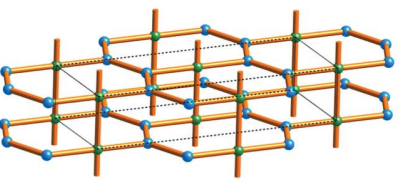

0

tfc, $\mathrm{Cmmm}$

Figure 6

Embeddings of the minimal nets with collisions derived from hms and tfc. The link shown in black has zero length for a full symmetry (given at the bottom of the figure) embedding. Symbols as in Fig. 3.

symmetric TPMS, is murky at best for the lower-symmetry variants, though it can be made rigorous with some effort (Schröder et al., 2003).

We can ask the inverse question: what is the surface associated with a given net? If we consider nodes of an embedding as small balls and links as small cylinders, it is clear that the surface of this assembly is a periodic surface unique to the net. But if one asks what is the balance surface separating two identical nets such as those of a tiling and its dual for a net with self-dual tiling the answer is less clear. A strategy to suggest an answer is as follows.

In the Dress approach (Dress, 1984) to describing tilings in terms of extended Schläfli symbols (Delaney-Dress symbols, or $D$ symbols), a tile is divided into simplicial chambers. In the three-dimensional case the vertices of the tetrahedral chambers are the center of a tile, the center of a face of that tile, the center of an edge of that face and a vertex of that edge. Fig. 7 shows such a division of the proper tile for the diamond net (dia). Consider now this space filling of tetrahedra as a tiling the dual tiling will have vertices on a surface intermediate between the original net (in this case dia) and its dual. Furthermore that surface will be tiled by a four-valent tiling in fact by quadrangles and larger polygons that form a net that we call the -t net, in this example dia-t (also known to the RCSR as fuf). The three-dimensional tiling of the -t net has two kinds of tile - tiles, with two large faces, that are topological prisms, and that correspond to edges of the original net, and tiles, with $z$ large faces, corresponding to $z$-coordinated vertices of the original net.

Recall that for a tiling of a surface of genus $g$ with $v$ vertices, $e$ edges and $f$ faces (tiles), $v-e+f=2-2 g$. For a four-valent tiling with $f_{i} i$-sided tiles, $e=2 v$ and $e=\sum(i / 2) f_{i}$. From this we

Figure 5

Embeddings of the minimal nets with collisions derived from cds. The link shown in black has zero length for a full symmetry (given at the bottom of the figure) embedding. Symbols as in Fig. 3. 


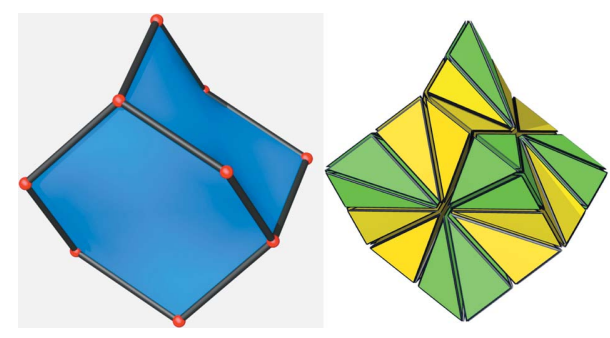

Figure 7

Left, a tile of the dia net. Right, the same divided into chambers.

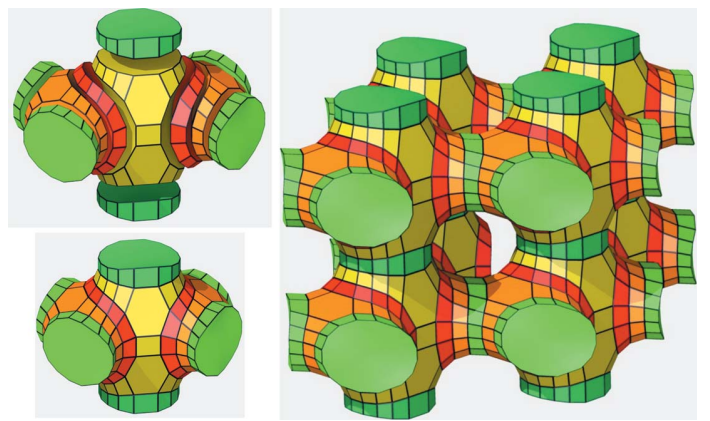

\section{Figure 8}

Left, tiles of the - $\mathbf{t}$ net for tfc. Right, an assembly of such tiles. Note how the tile (yellow) with four large faces and the two tiles with three large faces (orange) combine with two edge tiles (red) to form a larger tile with four orange and two yellow large faces. These are then linked by green edge tiles into a structure that is a slight distortion of the cubic $P$ surface.

find that $\sum(i-4) f_{i}=8 g-8$. Note that $f_{4}$ is unconstrained. For an MMS with $g=3$ and quadrangular and hexagonal tiles, $f_{6}=$ 8. In fact for the -t tiling of those nets that are the labyrinth graphs of the genus- 3 minimal surfaces the centers of the hexagons correspond to the flat points of that surface (see Figs. 8-10).

Fig. 8 shows part of a tiling for the tfc-t net. Note how the tiles for a 4-coordinated and two 3-coordinated vertices together with the tiles for their common edges merge to make a tile for a 6-coordinated vertex. The 6-coordinated assemblies then combine to form a surface with the same shape as the $P$ surface. This means that the tfa net could serve as the labyrinth graph of the $P$ surface as well as the pcu net. Thus the relation of nets to surfaces is many to one, as already indicated by Schröder et al. (2003). Fig. 9 shows how the tiles for tfa-t and ths-t similarly combine to give a surface that has the labyrinths of the $D$ surface.

Fig. 10 shows the tiling for srs-t. This illustrates a tiling of the $G$ surface and is shown compared with a proper tile of the srs net. It also illustrates that the surface is an inflated version of the net.

What of other tilings of minimal nets? Recall that the genus of a net is given by $1+e-v$. For a tiling of three-dimensional Euclidean space with $t$ tiles per repeat unit $v-e=t-f$ (Coxeter, 1973). But $t-f$ is equal to $v-e$ for the dual tiling.

\footnotetext{
$\overline{3}$ These correspond to the 12 distinct spanning trees that are subgraphs of the minimal net quotient graphs (Bonneau et al., 2004).
}

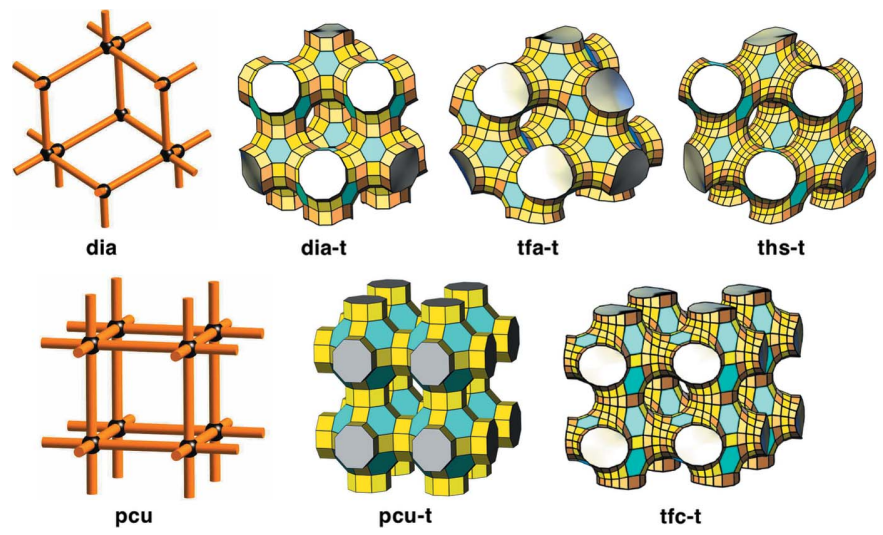

Figure 9

Tilings of the -t nets of pcu and dia and the three minimal nets not previously identified as labyrinth graphs of triply periodic surfaces. Note that pcu-t is the same as rho (Fig. 1).
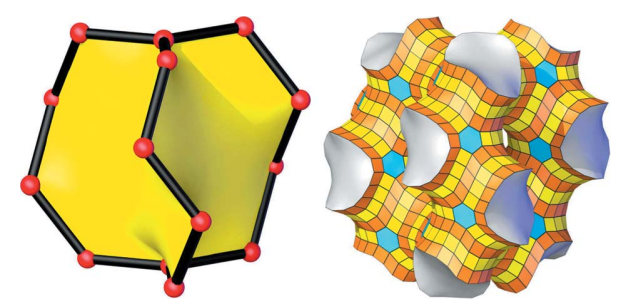

Figure 10

Left, a proper tile of the srs net. Right, part of the srs-t structure - a tiling of the $G$ surface. See also Fig. 12 .

Accordingly, the net of a dual of a tiling of a minimal net is another minimal net. Indeed, Bonneau et al. (2004) showed that there were 12 distinct ways $^{3}$ of dissecting a cube into smaller tiles and that all these tilings have duals which were tilings of one of the other minimal nets without collisions. However, there is a powerful theorem, valid only for genus-3 TPMSs, or MMSs. Meeks (1977) has proven that all flat points on MMSs are centers of inversion symmetry for the structure. This implies, in particular, that the labyrinths of such surfaces are related to each other via inversion symmetry; therefore their labyrinth graphs are necessarily congruent or related as mirror images and thus identical topologically. Accordingly the -t tilings, derived in turn from self-dual tilings, that we have described are the only cases to be considered.

We have now shown that all of the 15 minimal nets can serve as the labyrinth graph of one of the five known MMSs, and this was one of our main goals: to show that there are not necessarily any yet undiscovered MMSs despite the larger number of minimal nets than known MMSs. In $\$ 4$ we bolster this observation by more quantitative examination of the optimal surfaces separating pairs of identical minimal nets.

For higher-genus nets that do not have proper self-dual tilings one still generates an intermediate surface that may be a minimal surface. We illustrate this (Fig. 11) for the pair of nets bcu (the 8-coordinated net of the body-centered cubic lattice) and the 4-coordinated nbo net whose unique proper tilings are duals. The net illustrates bcu-t (necessarily the same, because of the duality, as nbo-t) that is a tiling of the inter- 

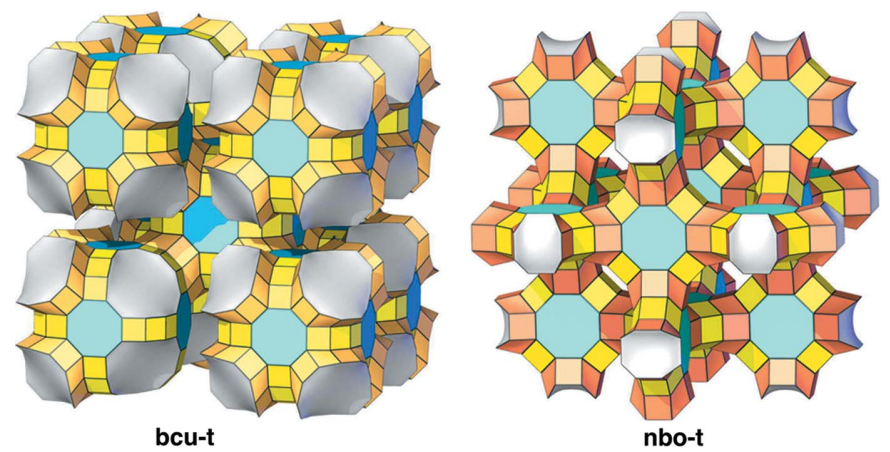

Figure 11

Two views of a tiling of the $I$-WP surface. The two periodic structures depicted combine to fill space. The 3-periodic nets bcu-t and nbo-t are identical.
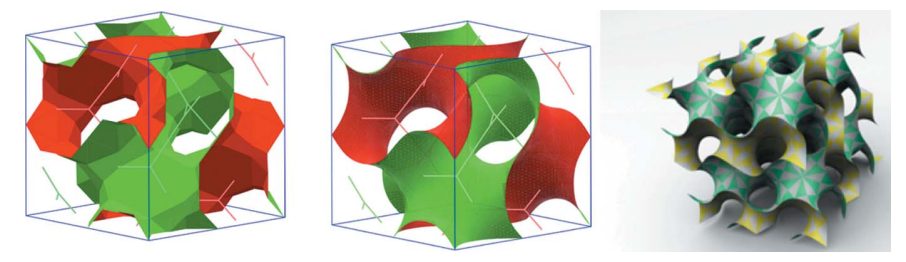

Figure 12

Left: the facetted sponge formed from Voronoi domains for a pair of (red and green) dual srs nets and (center) the relaxed surface generated by Surface Evolver. This surface is indistinguishable from the $G$ minimal minimal surface shown on the right.

mediate surface by quadrangles and octagons. The surface itself is the well known $I-W P$ minimal surface. Note that the inversion centers in this structure are at the nodes of the labyrinth graphs, in contrast to the case for genus 3 .

\section{Evolver experiments}

The resemblance of the '- $\mathrm{t}$ ' tilings above to genus-3 minimal triply periodic minimal surfaces is striking, though qualitative only. Can this be better quantified? A dual net pair can be used more directly to form approximations to TPMSs as follows. First, form Voronoi cells around each node of the embedded net combined with the net of its dual tiling. By construction, the interior of each cell is closer to the single internal net node than to any other node of the net pair. Next, remove any faces that are pierced by net links. The resulting structure is a topological sponge, with two open channels, made of planar faces. (It is often more convenient, and affords more rapid convergence, to place centers at the midpoints of the edges, generating a more finely facetted sponge.) Lastly, relax this facetted sponge to minimal surface area. In what follows we describe results obtained using the Surface Evolver program of Brakke (http://www.susqu.edu/brakke/evolver/), without changing the unit-cell shape or the topology of the sponge, to minimize the mean curvature of the surface. The resulting smooth surface then approximates a periodic minimal surface. We illustrate this procedure for a pair of dual srs nets in Fig. 12. We can compare the evolved surface to an exact embedding of the $G$ surface and there is no doubt that the solution converges to the gyroid TPMS. This is confirmed by plotting both surfaces together; provided a reasonable number of polygons are chosen in both cases they rapidly merge into a single surface, proving their equivalence with the numerical accuracy of the Evolver process.

A similar procedure for the dia, pcu, hms and cds nets (Fig. 13) results in evolved surfaces that contain straight lines lying in the surfaces. Those straight lines coincide with axes of twofold symmetry that exchange the (self-)dual net pair so are
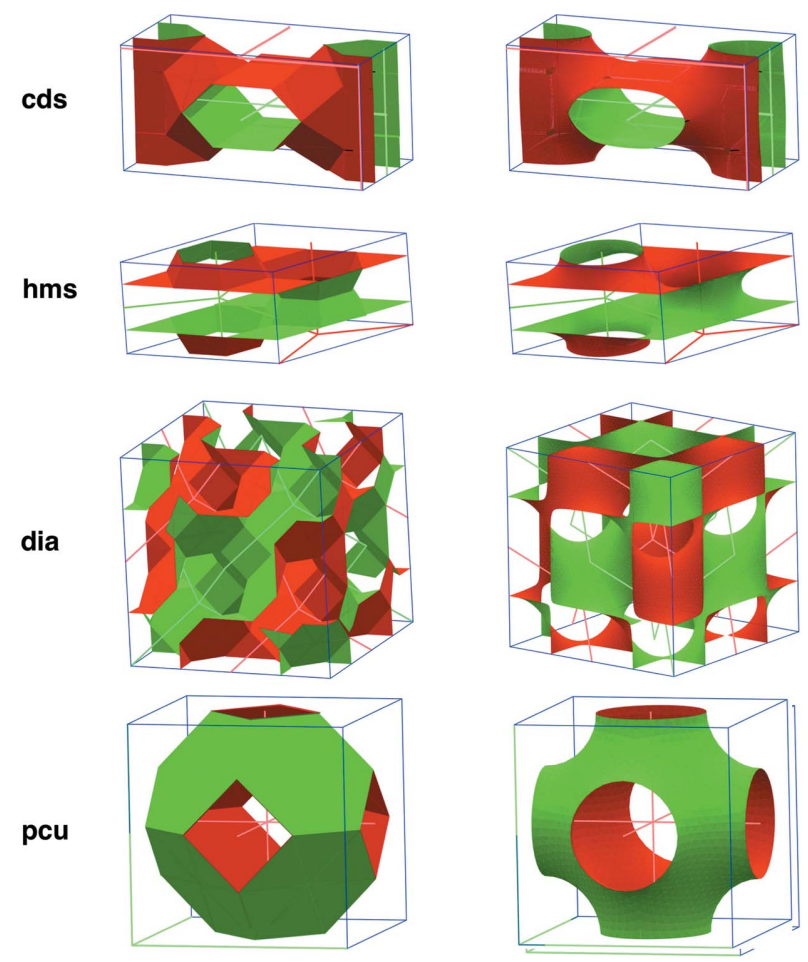

Figure 13

The facetted sponges (left) and the surfaces generated by Surface Evolver (right) for four 'parent' graphs.
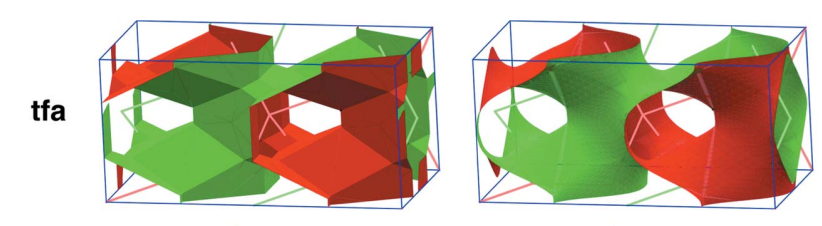

tfc
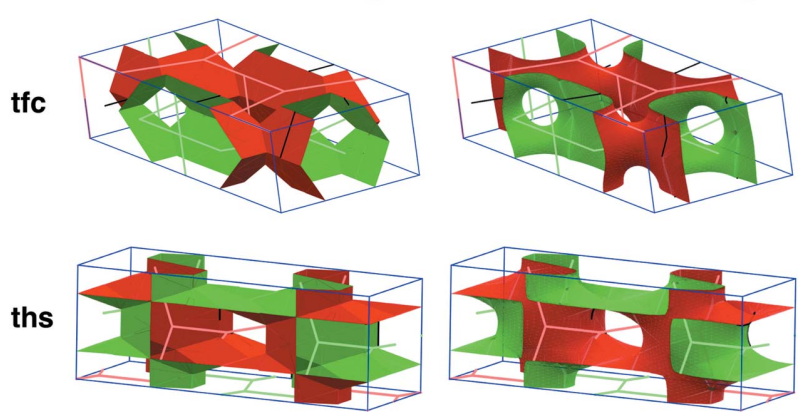

Figure 14

The facetted sponges (right) and the surfaces generated by the Evolver (right) for the nets tfa, tfe, and ths. 
necessarily present in the relaxed Voronoi cells. In all these cases except hms, the straight lines form closed skew polygons (with four, four and six sides, respectively). These polygons are precisely those found in the simpler genus-3 minimal surfaces and are signatures of those surfaces (see, for example, Fischer \& Koch, 1987; Koch \& Fischer, 1988). The hms example gives a stack of parallel hcb net layers. This skeleton defines the lines in the genus-3 $H$ minimal surface (Koch \& Fischer, 1988).

Similarly, the Evolver procedure applied to the nets tfa, ths and tfc (Fig. 14) generates surfaces with hyperbolic patches bounded by straight lines that can be used to identify the surface uniquely. The first pair of cases yields the skew pentagon characteristic of a tetragonal deformation of the $D$ surface, the genus-3 $t D$ surface (Fogden \& Hyde, 1992). ${ }^{4}$

Clearly, then, all the collision-free minimal nets yield known MMSs. The remaining eight graphs with collisions in their maximum-symmetry forms are likewise labyrinth graphs of the five known MMSs and all 15 minimal nets are accounted for.

MO'K is supported by the World Class University program (R-31-2008-000-10055-0) and by the US National Science Foundation, grant No. DMR 1104798.

\section{References}

Beukemann, A. \& Klee, W. E. (1992). Z. Kristallogr. 201, 37-51. Blatov, V. A., Delgado-Friedrichs, O., O'Keeffe, M. \& Proserpio, D. M. (2007). Acta Cryst. A63, 418-425.

Bonneau, C., Delgado-Friedrichs, O., O'Keeffe, M. \& Yaghi, O. M. (2004). Acta Cryst. A60, 517-520.

Chung, S. J., Hahn, Th. \& Klee, W. E. (1984). Acta Cryst. A40, 42-50.

Coxeter, H. S. M. (1973). Regular Polytopes. New York: Dover Publications.
Delgado-Friedrichs, O. (2005). Discrete Comput. Geom. 33, 6781 .

Delgado-Friedrichs, O. \& O'Keeffe, M. (2003). Acta Cryst. A59, 351360.

Delgado Friedrichs, O., O'Keeffe, M. \& Yaghi, O. M. (2003). Acta Cryst. A59, 22-27.

Dress, A. W. M. (1984). Algebraic Topology. Springer Lecture Notes in Mathematics, No. 1172, pp. 56-72. Berlin: Springer.

Eon, J.-G. (2007). Acta Cryst. A63, 53-65.

Eon, J.-G. (2011). Acta Cryst. A67, 68-86.

Fischer, W. \& Koch, E. (1987). Z. Kristallogr. 179, 31-52.

Fischer, W. \& Koch, E. (1989). Acta Cryst. A45, 726-732.

Fogden, A., Haeberlein, A. M. \& Lidin, S. (1993). J. Phys. I, 3, 23712385.

Fogden, A. \& Hyde, S. T. (1992). Acta Cryst. A48, 575-591.

Fogden, A. \& Hyde, S. T. (1999). Eur. Phys. J. B, 7, 91-104.

Grünbaum, B. (2003). Discrete and Computational Geometry: the Goodman-Pollack Festschrift, edited by B. Aronov, S. Basu, J. Pach \& M. Sharir, pp. 461-488. New York: Springer.

Han, L., Miyasaka, K., Terasaki, O. \& Che, S. (2011). J. Am. Chem. Soc. 133, 11524-11533.

Hyde, S. T. (1989). Z. Kristallogr. 187, 165-185.

Hyde, S., O'Keeffe, M. \& Proserpio, D. (2008). Angew. Chem. Int. Ed. 47, 7996-8000.

Koch, E. \& Fischer, W. (1988). Z. Kristallogr. 183, 129-152.

Meeks, W. H. III (1977). Bull. Am. Math. Soc. 83, 134-137.

Meeks, W. H. III (1990). Indiana Univ. Math. J. 39, 877-936.

Oguey, C. (1999). In Foam and Emulsions, edited by J. F. Sadoc \& N. Rivier. Dordrecht, Boston, London: Kluwer.

O'Keeffe, M., Peskov, M. A., Ramsden, S. J. \& Yaghi, O. M. (2008). Acc. Chem. Res. 41, 1782-1789.

Ramsden, S. J., Robins, V. \& Hyde, S. T. (2009). Acta Cryst. A65, 81108.

Schoen, A. H. (1970). NASA Technical Note, TN D-5541.

Schoen, A. H. (2012). Interface Focus 2, 529-538.

Schröder, G. E., Ramsden, S. J., Christy, A. G. \& Hyde, S. T. (2003). J. Eur. Phys. B, 35, 551-564.

Schwarz, H. A. (1890). Gesammelte Mathematische Abhandlungen. Berlin: Springer.

Weyhaupt, A. G. (2008). Pacific J. Math. 235, 137-171.

\footnotetext{
${ }^{4}$ The sponge formed from the tfa net pair relaxes in Evolver to a surface with patches bounded by lines that are not the full complement of those formed in the $t D$ surface. However, a simple argument proves that the surface with the full complement of lines must have a smaller area than that we repeatedly obtain numerically. The tfc net pair gives a relaxed surface whose patches are identical to those of an orthorhombic distortion of the $P$ surface, namely the $o P b$ surface (Fogden \& Hyde, 1992).
} 